\section{Aplicación de un nuevo programa de habilidades sociales a un grupo de adultos con discapacidad intelectual}

\author{
Implementation of a new social skills \\ training program for adults with intellectual \\ disabilities
}

\section{Resumen}

El presente estudio tiene como fin elaborar y aplicar un nuevo programa de entrenamiento para fomentar el uso de habilidades sociales (HHSS) en un grupo de personas adultas con discapacidad intelectual (DI). Contó con un diseño metodológico cuasi-experimental para probar la efectividad del programa. La muestra estuvo formada por $2 \mathrm{I}$ adultos con discapacidad intelectual, usuarios de una unidad de día con terapia ocupacional (Io participantes siguieron el programa y I I no). Las habilidades sociales fueron evaluadas por medio de una adaptación de la Escala de Habilidades Sociales de Gismero (2010). Los resultados ponen de manifiesto una mejoría significativa en las puntuaciones globales del grupo de tratamiento respecto al grupo contraste. De igual forma, se evidenció un incremento en las puntuaciones del grupo de tratamiento en cinco de las seis subescalas. Las mejoras detectadas resultan claves para el desarrollo individual de este grupo y se discuten las implicaciones de los resultados.

\section{Palabras clave}

Habilidades sociales, discapacidad intelectual, programa de entrenamiento, adultos.

\section{Abstract}

The present study has the purpose to develop and apply a new training program in order to promote the use of social skills in a group of adults with intellectual disabilities. It contains a quasi-experimental methodological design to prove the program effectiveness. The sample used consists of 2 I adults with intellectual disabilities, users of an occupational therapy day entity ( $\mathrm{s} \circ$ persons participated in the program and I I did not). The social skills were evaluated using an adjusted version of the Social Skills Scale Model of Gismero (2010). The outcomes of this study show a significant improvement in the overall score of the group included in the program in comparison with the rest of the group. Likewise, the group under the program obtained an increase in the scores within 5 out of 6 subscales evaluated. The identified improvements are key elements for the individual development of this group. The implications of the results are discussed.

\section{Keywords}

Social skills, intellectual disability, training program, adults.

\section{Fernanda Sequera \\ Fernández \\ <sequerafernanda@gmail.com>}

Fundación Síndrome de Down Madrid

\section{Eva M. Padilla-Muñoz \\ <evapadi@us.es>}

Universidad de Sevilla

\section{José Manuel Chirino Núñez}

$<$ psicologia.santiponce@pazbien. org $>$

Asociación Paz y Bien. Universidad de Sevilla

\section{José Antonio Pérez-Gil †}

Profesor titular del departamento de Psicología Experimental de la Universidad de Sevilla. In memoriam

Para citar:

Sequera, F. et al. (20I6): "Aplicación de un nuevo programa de habilidades sociales a un grupo de adultos con discapacidad intelectual". Revista Española de Discapacidad, 4 (2): 63-80.

Doi: <https://doi.org/IO. 5569/23405 I04.04.02.04>

Fecha de recepción: 2I-06-20I6 Fecha de aceptación: 02-I2-20I6 


\section{Introducción ${ }^{1}$}

La undécima edición de la American Association on Intellectual and Developmental Disabilities (AAIDD) (20II) ha continuado avanzando dentro del enfoque ecológico y del paradigma de apoyos que ya inició en las anteriores ediciones de I992 y 2002. En este marco, las habilidades sociales constituyen uno de los tres dominios de habilidades o conductas de adaptación, junto con las conceptuales y las prácticas.

La evaluación de la conducta adaptativa comenzó a proliferar a partir de los años ochenta y de forma gradual se empezó a tener en cuenta para la planificación de la intervención psicológica (Verdugo y Gutiérrez Bermejo, 2009), con el objetivo de lograr un nivel óptimo de funcionamiento, especialmente en la población de personas con discapacidad intelectual. Bajo esta premisa, se sustenta la necesidad de realizar programas específicos -ad hoc- para entrenar HHSS en la población de personas adultas con DI.

Vicente Caballo es referencia obligada en nuestro contexto para hablar de HHSS, quien las definió como aquellas conductas que se producen en contextos interpersonales, y que son reflejo de sentimientos, actitudes, derechos y opiniones de la persona (I993). No obstante, no existe todavía un consenso aceptado sobre qué aspectos deben incluirse o no en este constructo. Así, mientras de un lado se pone el énfasis en que son conductas dirigidas a la solución de problemas (es el caso de Wilkins y Matson, 2009) en población adulta con autismo y/o DI, para otros, el entrenamiento de estas habilidades debe dirigirse a incrementar la aceptación social

I. Agradecemos especialmente a las personas (adultos con discapacidad intelectual) que han dado su consentimiento para participar en el programa de entrenamiento, así como a todos aquellos técnicos (auxiliares, educadores, psicólogos...) de la Asociación Paz y Bien en Santiponce (Sevilla) que también facilitaron e hicieron posible este trabajo. y la calidad de vida del individuo (Bellack, I983, o.c. en Arias et al., 2014).

En este trabajo nos interesa especialmente el matiz de que son conductas entrenables. Con todo, actualmente, las HHSS pueden ser entendidas, de forma genérica, como aquellas conductas necesarias para interactuar y relacionarse con los demás de manera efectiva y mutuamente satisfactoria, y se caracterizan por ser conductas adquiridas a través del aprendizaje; presentar componentes motores, emocionales, cognitivos y comunicativos; ser respuestas específicas a situaciones concretas y ponerse en juego en contextos interpersonales (Rosales et al., 2013). Por tanto, estas habilidades constituyen una herramienta de gran utilidad para el desenvolvimiento diario, y gracias a ellas las personas pueden mejorar su capacidad para relacionarse y cubrir mejor sus necesidades; en definitiva, favorecen la calidad de vida.

Pero no sólo es importante disponer de un repertorio de conductas socialmente útiles, sino saber ponerlas en práctica en la situación adecuada, lo que denominamos competencia social (Gutiérrez Bermejo y Prieto, 2002). Es, principalmente, esta falta de adecuación de las conductas al contexto la principal carencia observada en las personas con limitaciones cognitivas.

La mayoría de los estudios revelan que los individuos con DI podrían hacer progresos en su nivel de ajuste general a medida que se instalan en la vida adulta; sin embargo, muy a menudo se comportan de manera inapropiada en las situaciones sociales y son rechazados al llegar a esta etapa. Se muestran socialmente incompetentes e incapaces de conseguir cambios en sus vidas y de responder de manera efectiva en la sociedad. Para Umadevi y Sukumaran (20I2), estas inadecuadas conductas sociales de las personas con DI podrían ser el resultado de percepciones incorrectas de ciertas situaciones sociales. 
En la pasada década, algunos autores, como Elksnin y Elksnin (200I), ya destacaron que los déficits específicos en HHSS y las consecuencias en el comportamiento son predictores de dificultades posteriores como desadaptación en el empleo. En la misma dirección, Bremer y Smith (2004) argumentan que parte de los problemas que incorpora el déficit en estas habilidades se traducen en conductas oposicionistas, problemas conductuales, influencias negativas de grupos de pares, abuso de drogas o dificultades familiares, entre otras. Matson y Boisjoli (2008) añaden que la ausencia de habilidades sociales puede suponer un importante impedimento para la vida independiente y favorece la aparición de psicopatología comórbida en este colectivo.

Gutiérrez Bermejo y Prieto (2002), en nuestro contexto y tras la evaluación de HHSS utilizando componentes de conductas verbales y no verbales en una muestra de más de 650 usuarios de centros de personas con discapacidad, ponen de manifiesto que el entrenamiento en HHSS debe ser uno de los aspectos clave en la intervención con personas con DI, tanto por ser deficitarias en esta población como por su relevancia para una integración social con éxito.

No obstante, si partimos de la idea de que las HHSS son conductas adquiridas a través del aprendizaje, gran parte de estas limitaciones y consecuencias adversas recogidas en la literatura se pueden minimizar por medio de un correcto entrenamiento en la conducta social de la persona con DI en un contexto interpersonal significativo. Además, para Gutiérrez Bermejo y Prieto (2002), la adquisición de este tipo de habilidades debe servir como amortiguador de una amplia gama de situaciones conflictivas que tienden a ser comunes en esta población. Asimismo, el entrenamiento de las HHSS favorece la integración y desinstitucionalización exitosa de la persona con DI, resulta crucial para evitar la aparición de problemas de comportamiento y para prevenir la aparición de problemas psicológicos y de abusos sexuales. Por tanto, las ventajas de su entrenamiento se unen al posible fracaso derivado del déficit en las relaciones sociales en este grupo poblacional. Del mismo modo, para Ruiz (2007) resulta de especial relevancia el entrenamiento en HHSS para mejorar el grado de inclusión social de las personas con síndrome de Down, enfatizando este autor que estas habilidades se pueden mejorar y potenciar a través de experiencias de aprendizajes adecuados y adaptados al contexto donde se desenvuelve la persona.

Los programas para el entrenamiento de HHSS dirigidos a la población general han proliferado de manera notable en las últimas décadas. No obstante, estos no pueden dar respuesta a las necesidades específicas que presentan las personas con DI en los diferentes contextos en los que se desenvuelven. Por ello, la información proveniente de la evaluación individualizada (o en pequeño grupo) en dichos entornos se hace muy necesaria para elaborar programas específicos que permitan mejorar el nivel de competencia social de estos individuos, permitiendo contribuir a una verdadera planificación centrada en la persona (Verdugo y Gutiérrez Bermejo, 2009).

Sobre la eficacia del entrenamiento en habilidades sociales en esta población, Hair et al. (2002) observaron que los adolescentes con discapacidad que tienen buenas destrezas sociales, particularmente en las áreas de resolución de conflictos, intimidad emocional y el uso de conductas prosociales, aumentan su probabilidad de ser aceptados por los demás, formando buenos lazos de amistades, mejores relaciones con sus padres y demás personas, resaltando el carácter sostenido de las habilidades sociales y la importancia de seguir entrenándolo a lo largo de la vida, realizando adaptaciones específicas según el momento en que se encuentre y el contexto donde se desenvuelva: ambiente laboral, comunitario y/o familiar.

O'Reilly et al. (2004) compararon la efectividad del entrenamiento en HHSS en un grupo de cinco adultos con DI que vivían en pisos tutelados de la comunidad. En la misma línea, Anderson y Kazantzis (2008), en un estudio de caso múltiple, aplican un programa específico 
sobre resolución de problemas sociales a tres adultos con DI usuarios de un centro comunitario. En ambos trabajos, los resultados ponen de manifiesto mejoras en todos los participantes y mantenimiento de las habilidades adquiridas un mes después del entrenamiento.

Más recientemente, Lachavanne y Barisnikov (2013) aplicaron un programa para la reeducación de las habilidades socioemocionales, con pre y post, a un grupo de I7 adultos con DI y observaron cambios significativos positivos tras la aplicación del programa. También con un diseño pre-post, Liu et al. (2013) desarrollan un programa específico de formación laboral para un grupo de I 4 personas con discapacidad intelectual y autismo, que incluye entrenamiento específico en HHSS Los resultados tras la aplicación del programa son satisfactorios.

En nuestro ámbito, contamos con escasas experiencias de programas de entrenamiento específicos sobre HHSS dirigidos a personas adultas con DI, que hayan sido suficientemente controlados y con datos sobre evaluación de resultados. Destacamos el trabajo de Boluarte et al. (2006), en el que se valora la elaboración y aplicación de dos programas. El primero de I 8 sesiones para jóvenes con DI leve, donde se trabajan áreas como: conductas no verbales, habilidades de planificación, HHSS básicas (hábitos de cortesía), habilidades conversacionales, reconocimiento y expresión de emociones, expresión de halago, autocontrol, asertividad y manejo de la crítica, entre otros. El segundo, para jóvenes con DI moderada, consta de I 2 sesiones de trabajo en las que se entrenan: integración familiar, conductas no verbales, HHSS básicas, reconocimiento y expresión de emociones, dar y recibir halagos y prevención del peligro (rechazo de peticiones). Los autores observaron que tras la implementación de estos programas mejoraron significativamente las habilidades de comunicación verbal y no verbal de ambos grupos de jóvenes. Por su parte, en el trabajo de Martínez et al. (2004), aplicando un programa similar a una población con trastornos mentales graves (sin DI pero con otras limitaciones mentales graves), también se obtienen resultados positivos tras la intervención.

De manera general, se concluye que es recomendable reforzar la competencia social de las personas con limitaciones cognitivas y potenciar sus áreas de participación dentro de la sociedad, lo que facilitará su integración en los distintos contextos.

No obstante, a pesar de los beneficios de la aplicación de estos programas, todavía existen contadas experiencias de implementación de programas específicos en contextos comunitarios que permitan mejorar el grado de capacitación social de estos individuos y, lo que es más importante, pocas de estas experiencias cuentan con un diseño metodológico adecuado, con medidas pre y post y grupo de contraste.

El presente estudio tiene como objetivo elaborar y aplicar un nuevo programa de entrenamiento para fomentar el uso de las HHSS en un grupo de personas adultas con DI para optimizar su nivel de funcionamiento general.

Una de sus principales aportaciones es contar con un diseño metodológico adecuado para poner a prueba la efectividad del programa de entrenamiento, realizando una evaluación previa y otra evaluación tras la finalización del mismo, y comparando dichos resultados con los obtenidos por un grupo de contraste.

Como hipótesis general, podemos establecer que se producirán cambios positivos, es decir, un incremento en las competencias sociales medidas (autoexpresión en situaciones sociales, defensa de los propios derechos como consumidor, expresión de enfado o disconformidad, decir no y cortar interacciones, hacer peticiones e iniciar interacciones positivas con el sexo opuesto), tras la aplicación del nuevo programa de HHSS en el grupo de adultos con DI que participa, mientras que en el grupo de no participantes, las puntuaciones permanecerán estables antes y después. 


\section{Método}

Este programa de entrenamiento va dirigido al universo de adultos con DI usuarios de una unidad de estancia diurna con terapia ocupacional de la Comunidad Autónoma de Andalucía. La población en la que nos centramos fue la de todos aquellos usuarios que estaban en lista de espera para recibir un programa de intervención en HHSS, 65 de un total de $\mathrm{I} 50$ casos atendidos, y que asistían a la misma unidad de estancia diurna con terapia ocupacional. La muestra final fue establecida por conveniencia, y estuvo formada por $2 \mathrm{I}$ personas usuarias de dicha unidad, mayores de I 8 años con DI. Específicamente, el rango de edad quedó comprendido entre los I 9 y 58 años de edad y la distribución por sexos fue de I 3 hombres $(62 \%)$ y de 8 mujeres (38\%).

Se seleccionaron dos grupos: grupo de tratamiento (GT), con participación en el programa de entrenamiento, y grupo de contraste (GC), de características similares, pero sin participación en el programa. Las condiciones para la selección de la muestra final y los criterios de inclusión y exclusión se exponen brevemente:

- Todas las personas participantes asistían diariamente a la misma unidad de estancia diurna con terapia ocupacional de la provincia.

- Todos tenían en su planificación personal, como objetivo a trabajar para la mejora de su calidad de vida, el entrenamiento en HHSS y en asertividad.

- Ninguno de ellos había participado previamente en un programa de entrenamiento de HHSS organizado desde el área de psicología.

- La variable sexo debía quedar convenientemente balanceada en los grupos, así como la representación de diferentes rangos de edad.

- El nivel cognitivo de los participantes fue similar en ambos grupos (con grado total de discapacidad entre un $65 \%$ y un $88 \%$ según diagnóstico del centro de valoración y orientación.)

- Como criterio de inclusión, todos los participantes debían tener adquiridas las habilidades comunicativas necesarias para expresar y comprender instrucciones sencillas.

Teniendo en cuenta todo lo anterior, los criterios seguidos para disponer a los diferentes participantes en los grupos de contraste $(\mathrm{GC})$ y de tratamiento (GT), respondieron meramente a criterios organizativos del servicio, conformando en primer lugar el GT y a partir del mismo el GC, cuidando que fueran similares en la distribución del sexo, la edad, el grado total de discapacidad (entre un $65 \%$ y un $88 \%$ ) y los diagnósticos (el $90 \%$ sin etiología filiada).

\section{- Diseño}

Siguiendo a León y Montero (I997), en el presente estudio se utilizó un diseño pre-post con grupo de tratamiento cuasi experimental:

$$
\begin{aligned}
& \mathrm{O}_{1} \mathrm{XO}_{2} \\
& \mathrm{O}_{\mathrm{I}} \mathrm{CO}_{2}
\end{aligned}
$$

Como variable dependiente se tomó la puntuación total en la Escala Adaptada de Habilidades Sociales de Gismero (EAHS) (20I0). La variable independiente quedó precisada por la aplicación o no de un programa de entrenamiento en HHSS a un grupo de personas adultas con DI. Asimismo, para aumentar el control de las variables extrañas e incrementar el grado de validez interna del estudio, se utilizó un diseño equivalente con grupo de contraste (GC).

Este diseño resulta ser el más adecuado según las características de la investigación, ya que se ha trabajado con grupos hechos (participantes ya preseleccionados) y no ha sido posible la asignación aleatoria de los sujetos a uno u otro grupo (condición imprescindible en los diseños experimentales) (Morales, 20I3). Para cumplir 
con los criterios de cuasi-experimentalidad, el GC se constituyó a partir de un grupo de participantes al que se le aplica el valor nulo de la variable independiente; en este caso, el programa de entrenamiento en HHSS.

Fue importante asegurarse de que ambos grupos fuesen lo más similares posible en las variables que se querían controlar. La evaluación previa a la aplicación del programa nos permitió comprobar la semejanza en la variable dependiente en ambos grupos lo que nos permitió utilizar el término "cuasi". Shaughnessy et al. (2007) indican que sólo se puede dar este tipo de diseño si existe un grupo semejante al grupo de tratamiento que funcione como grupo de comparación y si hay oportunidad de obtener medidas pretest y postest de los integrantes de ambos grupos, tal y como se hizo en este estudio.

\section{- Instrumentos}

Los tres métodos más comunes para evaluar las HHSS son las observaciones conductuales, el role-playing y las escalas (Bielecki y Swender, 2004). Si bien, los autoinformes y las escalas poseen una utilidad limitada porque no ofrecen datos muy específicos sobre los requisitos del entrenamiento de las personas con déficit de estas habilidades, sí nos proporcionan información de carácter general acerca de la conducta de una persona o de un pequeño grupo en situaciones interpersonales. Además, han demostrado ser una de las fuentes de datos más útiles para la evaluación general de una intervención; así como una de las formas más viables de evaluar el efecto y la generalización de la misma (Kelly, 2002).

Para la evaluación de las HHSS, en el pre y post del GT y del GC, se decidió aplicar la Escala de Habilidades Sociales de Gismero (2010), por su especificidad para medir conducta asertiva o HHSS, su brevedad y la disposición de datos normativos en muestras españolas. La Escala está formada por 33 ítems que se dividen en seis subescalas o factores: I "Autoexpresión en Situaciones Sociales”, II “Defensa Derechos como Consumidor", III "Expresión de Enfado o Disconformidad”, IV “Decir no y cortar Interacciones", V "Hacer Peticiones” y VI "Iniciar Interacciones Positivas con el Sexo Opuesto”.

Las razones para elegir este instrumento fueron: la posibilidad de obtener un índice global de HHSS o aserción, que ofrece información específica sobre dónde se localizan los puntos que necesitan ser más potenciados y la facilidad de aplicación. En relación con la fiabilidad y validez de la Escala, se han calculado los coeficientes de fiabilidad mediante el procedimiento alfa de Cronbach en sus tres versiones, en las dos versiones experimentales y en la definitiva. Y la fiabilidad se mantiene casi idéntica en las tres. Se adopta la versión de 33 ítems como definitiva, con una fiabilidad de $\circ, 88$. No obstante, para poder aplicarla en el presente estudio y, dadas las características de la población, fue necesario realizar una adaptación de esta Escala para permitir que los contenidos fueran más accesibles a los usuarios participantes.

Entre las adaptaciones de la Escala fue necesario introducir ejemplos en los ítems que representaban mayor complejidad. Otro elemento modificado fue la escala de respuesta, que podía generar confusión a los participantes. Conservamos las cuatro opciones de respuesta, para no alterar la esencia de la escala, pero aplicamos un lenguaje más sencillo y comprensible: totalmente en desacuerdo, desacuerdo, de acuerdo y totalmente de acuerdo. La forma de responder también se modificó, al colocar en cada opción de respuesta, en vez de círculos, iconos con expresiones según la opción de respuesta, como ayuda visual. Se presentaron para cada ítem cuatro iconos relacionados con la escala de respuesta. Desde iconos de pequeñas dimensiones con expresión triste para la opción totalmente en desacuerdo hasta iconos de grandes dimensiones, con expresión contenta para la opción totalmente de acuerdo. Sin duda, todas estas adaptaciones facilitaron el proceso de evaluación. En el momento de aplicación todos los participantes contaban con los mismos ejemplos y formato en las opciones de respuesta (ver Anexo I, ejemplos de ítems adaptados). 


\section{- Procedimiento}

El programa de entrenamiento en HHSS fue aplicado a un grupo de adultos con DI que asistían a una unidad de estancia diurna con terapia ocupacional. Cada participante en el estudio contaba con un Programa Personalizado de Apoyos (PPA), diseñado por el equipo psicoeducativo del servicio y acorde a los intereses y necesidades individuales de la persona usuaria con DI.

Dicho programa es elaborado, implantado y evaluado siguiendo las directrices del Protocolo de Atención Personalizada editado por la Consejería de Igualdad y Bienestar Social de la Junta de Andalucía (VV.AA., 20Io). Cada PPA se convierte en un documento de trabajo individual donde cada sujeto participante, así como los familiares de éste, firman conocer y estar de acuerdo con los objetivos, la metodología y las actividades propuestas.

Nos cercioramos de que todos los participantes, pertenecientes tanto al GC como al GT, contaban con un PPA entre cuyos objetivos figuraba mejorar su repertorio de HHSS o su relación interpersonal. Además, se constató que ninguno de los integrantes del GT y del GC había participado con anterioridad en una acción formativa o programa de HHSS organizado y puesto en marcha desde el área de psicología del servicio.

En la unidad de estancia diurna con terapia ocupacional se llevan a cabo programas de entrenamiento grupales con periodicidad variable y carácter cíclico, seleccionando los integrantes de cada grupo de trabajo en función de las necesidades detectadas en los mencionados PPA. Dichas acciones grupales están en conocimiento y cuentan con la aprobación explícita del comité de ética de la entidad. Asimismo, el citado comité certificó por escrito que el programa de habilidades sociales fue debidamente revisado, después de comprobar que los procedimientos utilizados eran acordes con los principios éticos de la entidad.
El programa de entrenamiento aplicado al grupo de participantes que formó el grupo de tratamiento estuvo constituido por nueve sesiones de trabajo, dos de ellas dedicadas a la evaluación y las otras siete a la implementación y ejecución del mismo. La duración fue de dos meses aproximadamente, a razón de una sesión semanal de una hora y I 5 minutos. En concreto, las sesiones se llevaron a cabo los lunes entre las I 5:I 5 y las I 6:30 horas.

El presente programa estuvo configurado por una serie de actividades agrupadas en diferentes sesiones; cada sesión tenía asignado distintos objetivos específicos. Siguiendo las recomendaciones de Kelly (2002) para el entrenamiento de las HHSS en grupo, en cada sesión se incorporan instrucciones y explicación del componente seleccionado para ese día, modelado, ensayo de conducta, reforzamiento y feedback. De forma específica, para este programa, las sesiones fueron elaboradas según las necesidades principales que se detectaron a partir de la aplicación de la Escala Adaptada de Habilidades Sociales de Gismero.

Principalmente, nos basamos, en la propuesta de programa de entrenamiento sobre HHSS de Roca (2007) y seguimos una línea de trabajo similar a la que se venía desarrollando en el centro. La propuesta de cada sesión queda recogida en la tabla $\mathrm{I}$.

Los objetivos de cada sesión se elaboraron en relación con las dimensiones que necesitaban mayor refuerzo según la evaluación previa de la EAHS. El componente con mayores limitaciones fue el denominado "Autoexpresión en situaciones sociales", seguido de la "Defensa de los propios derechos como consumidor", la "Expresión de dnfado y de disconformidad", el "Inicio de interacciones positivas con el sexo opuesto", "Hacer peticiones” y, por último, "Decir no y cortar interacciones". 
Tabla I. Resumen de las sesiones del programa de entrenamiento en HHSS

\begin{tabular}{|c|c|c|c|}
\hline $\begin{array}{l}N^{\circ} \text { de } \\
\text { sesión }\end{array}$ & Objetivos específicos & Actividades & Metodología \\
\hline Sesión 1 & - Aplicar EAHS al GC y GT (Pre). & $\begin{array}{l}\text { - Sesión de evaluación de las } \\
\text { HHSS por medio de la EAHS. }\end{array}$ & $\begin{array}{l}\text { Explicación de la escala } \\
\text { por medio de ejemplos } \\
\text { en grupos de cinco } \\
\text { personas aprox. }\end{array}$ \\
\hline Sesión 2 & $\begin{array}{l}\text { - Crear conciencia de grupo. } \\
\text { conplorar sobre los } \\
\text { grupo sobre HHSS. } \\
\text { - Acercar a los participantes al } \\
\text { concepto de HHSS. } \\
\text { Introducir al grupo los estilos } \\
\text { de comunicación. }\end{array}$ & 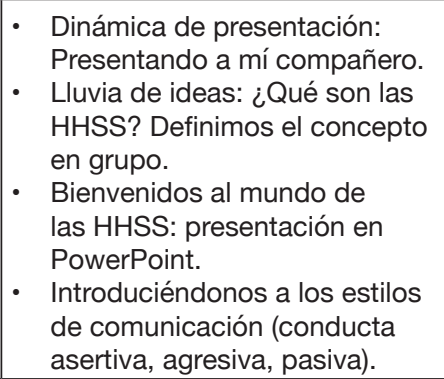 & $\begin{array}{l}\text { Trabajo en parejas, } \\
\text { lluvia de ideas, role } \\
\text { playing, preguntas de } \\
\text { comprobación. }\end{array}$ \\
\hline Sesión 3 & $\begin{array}{l}\text { Recordar conceptos y } \\
\text { ejemplos de los estilos de } \\
\text { comunicación (agresivo, } \\
\text { pasivo y asertivo). } \\
\text { Trabajar aspectos } \\
\text { relacionados con la } \\
\text { comunicación no verbal (CNV). }\end{array}$ & $\begin{array}{l}\text { - Recordamos conceptos de la } \\
\text { semana anterior. } \\
\text { - Conociendo qué es el lenguaje } \\
\text { no verbal. } \\
\text { Trabajamos la comunicación } \\
\text { no verbal por medio de } \\
\text { cómics. } \\
\text { - Entrevista y observadores. }\end{array}$ & $\begin{array}{l}\text { Role playing, mímica, } \\
\text { material visual (cómics) y } \\
\text { entrevista. }\end{array}$ \\
\hline Sesión 4 & $\begin{array}{l}\text { Recordar los elementos de la } \\
\text { CNV. } \\
\text { - Trabajar sobre la } \\
\text { comunicación verbal (CV). }\end{array}$ & 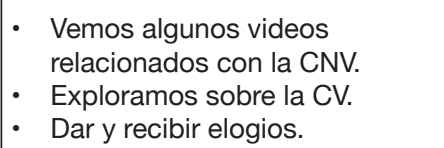 & $\begin{array}{l}\text { Vídeos, discusión grupal, } \\
\text { role playing. }\end{array}$ \\
\hline Sesión 5 & $\begin{array}{l}\text { - Comprender lo que es la } \\
\text { comunicación eficaz. } \\
\text { - Finalizar conversaciones de } \\
\text { forma eficaz. }\end{array}$ & $\begin{array}{l}\text { Role play: aprendemos a } \\
\text { finalizar conversaciones. } \\
\text { Entrevista: trabajando la } \\
\text { comunicación eficaz. }\end{array}$ & $\begin{array}{l}\text { Vídeos, discusión grupal, } \\
\text { role playing, entrevista. }\end{array}$ \\
\hline Sesión 6 & $\begin{array}{l}\text { Conocer formas asertivas de } \\
\text { hacer y rechazar peticiones. }\end{array}$ & $\begin{array}{l}\text { - ¿Cómo debemos hacer y } \\
\text { rechazar peticiones? } \\
\text { Qué técnicas podemos utilizar } \\
\text { para rechazar peticiones. Lo } \\
\text { ponemos en práctica. }\end{array}$ & $\begin{array}{l}\text { Modelado, role playing, } \\
\text { trabajo grupal. }\end{array}$ \\
\hline Sesión 7 & $\begin{array}{l}\text { Fomentar el uso de solución } \\
\text { de conflictos interpersonales. } \\
\text { - Aprender cómo solucionar } \\
\text { problemas. }\end{array}$ & $\begin{array}{l}\text { - "Me olvidé del dinero". } \\
\text { - "El naufragio". }\end{array}$ & $\begin{array}{l}\text { Debate, trabajo grupal, } \\
\text { lluvia de ideas. }\end{array}$ \\
\hline Sesión 8 & $\begin{array}{l}\text { - Introducir al grupo el concepto } \\
\text { de empatía. } \\
\text { - Comprobar los conocimientos } \\
\text { adquiridos por el grupo a lo } \\
\text { largo del programa. }\end{array}$ & $\begin{array}{l}\text { - "Cómo crees que se sentiría } \\
\text { sí..." } \\
\text { - Vídeos. } \\
\text { - Juego de fichas. }\end{array}$ & $\begin{array}{l}\text { Role playing, actividad } \\
\text { grupal, vídeos, ronda de } \\
\text { preguntas y respuestas. }\end{array}$ \\
\hline Sesión 9 & $\begin{array}{l}\text { - Aplicar la EAHS a GC y GT } \\
\text { (Post) }\end{array}$ & $\begin{array}{l}\text { - Sesión de evaluación: postest } \\
\text { de las HHSS por medio de la } \\
\text { EAHS. }\end{array}$ & $\begin{array}{l}\text { Explicación de la escala } \\
\text { por medio de ejemplos } \\
\text { en pequeños grupos de } \\
\text { aproximadamente cinco } \\
\text { personas. }\end{array}$ \\
\hline
\end{tabular}

Fuente: elaboración propia. 
Debemos mencionar, que aunque las sesiones no fueron programadas para trabajar cada subescala de forma específica, sí se tuvieron en cuenta de forma transversal, es decir, los ejercicios prácticos y situaciones planteadas a lo largo del programa guardaban relación con los propuestos en EAHS. A modo de ejemplo ilustrativo, y con la idea de aportar una descripción más detallada, destacamos algunas situaciones trabajadas sobre cada una de las subescalas:

- Subescala I: Autoexpresión en situaciones sociales.

Situación: A veces evito ciertas reuniones sociales por miedo a hacer o decir alguna tontería.

- Subescala 2: Defensa de los propios derechos como consumidores.

Situación: Si al llegar a mi casa encuentro un defecto en algo que he comprado, voy a la tienda a devolverlo.

- Subescala 3: Expresión de enfado o disconformidad.

Situación: Cuando algún amigo expresa su opinión con la que estoy muy en desacuerdo, prefiero callarme a manifestar abiertamente lo que yo pienso.

- Subescala 4: Decir no y cortar interacciones. Situación: Hay determinadas cosas que me disgusta prestar, pero si me las piden, no sé cómo negarme.

- Subescala 5: Hacer peticiones. Situación: Si en un restaurante no me traen la comida como la había pedido, llamo al camarero y pido que me la haga de nuevo.

- Subescala 6: Iniciar interacciones positivas con el sexo opuesto.

Situación: Soy incapaz de pedir a alguien una cita.

Cabe destacar que a lo largo del programa los participantes se mostraban motivados, participativos y con una buena aceptación de las dinámicas propuestas. Específicamente, manifestaban un mayor interés en las actividades más vivenciales y prácticas (role playing, visionado de vídeos, debates, etc.) disminuyendo ligeramente el nivel de atención cuando se abordaban aspectos de carácter más teórico.

El programa de entrenamiento en HHSS fue diseñado-ad hoc- para trabajar las citadas dimensiones. Por su parte, los participantes del GC no fueron objeto de ningún tipo de entrenamiento. Aunque desde el área de psicología de los servicios de día, y de forma transversal a otras actividades, se desarrollan programas psicoeducativos de diferente índole: técnicas de relajación, inteligencia emocional y estimulación cognitiva entre otros. En este caso, ninguno de los participantes en el estudio, ni GC ni GT, formaron parte de estas acciones formativas en el periodo en que duró la aplicación del programa. En cambio, sí continuaron trabajando en sus tareas diarias según la dinámica del centro, es decir, actividades relacionadas con el manipulado y el ajuste personal según correspondiese, asegurándonos de que ninguna de estas acciones interfería en las actividades derivadas del programa.

Dos meses después, y una vez finalizado el programa de entrenamiento en HHSS, tanto el GC como el GT fueron evaluados de nuevo con la Escala Adaptada de Habilidades Sociales de Gismero para valorar su efecto. Dicho programa fue diseñado, puesto en práctica y evaluado por el primer y el tercer autor de este trabajo.

De todos los métodos de análisis posibles para realizar el contraste de medias, se seleccionó $t$ de Student, ya que a pesar del reducido tamaño de la muestra es la opción más utilizada cuando el tamaño de los grupos es similar y las varianzas no son distintas (Morales, 20I3).

\section{Resultados}

Presentamos los resultados más relevantes agrupados en dos apartados. En primer lugar, los datos relativos a la comparación entre los grupos para cada una de las subescalas del 
instrumento utilizado y, seguidamente, el análisis de las puntuaciones globales. En ambos casos, se aportan las puntuaciones de la evaluación previa (Pre) y de la posterior (Post) a la aplicación del programa.

\section{- Análisis de las subescalas de la EAHS:}

Inicialmente, se llevaron a cabo comparaciones de media entre los grupos GT y GC antes del inicio del programa (Pre). Los resultados obtenidos tras el análisis se reflejan en la tabla 2.
Se puede observar que no existen diferencias estadísticamente significativas entre las respuestas de ambos grupos en cada una de las subescalas, aceptándose de esta manera que ambos grupos tienen puntuaciones globales en HHSS similares en el Pre.

Seguidamente, se muestran los resultados obtenidos tras comparar las puntuaciones de los dos grupos tras la aplicación del programa. Para ello se ha aplicado la prueba $t$ para muestras relacionadas en cada una de las subescalas (ver tabla 3 ).

Tabla 2. Comparación de las medias pretest de la evaluación por subescala del GT y GC ( $t$ de Student)

\begin{tabular}{|l|c|c|c|c|c|}
\hline & \multicolumn{4}{|c|}{ Pretest } \\
\hline Subescala & \multicolumn{4}{|c|}{ GT (N=10) GC (N=11) } \\
\hline I: Autoexpresión en situaciones sociales & Media & SD & Media & SD & Sig. \\
\hline II: Defensa de los propios derechos como consumidor & 17,80 & 3,94 & 15,18 & 3,65 & NS \\
\hline III: Expresión de enfado o disconformidad & 12,90 & 3,18 & 12,64 & 4,08 & NS \\
\hline IV: Decir no y cortar interacciones & 8,50 & 3,31 & 9,91 & 3,39 & NS \\
\hline V: Hacer peticiones & 11,90 & 3,21 & 14,27 & 4,10 & NS \\
\hline VI: Iniciar interacciones positivas con el sexo opuesto & 14,00 & 2,35 & 10,50 & 4,16 & NS \\
\hline
\end{tabular}

NS: No significación.

Fuente: elaboración propia.

Tabla 3. Comparación de las medidas postest de la evaluación por subescalas del GT y GC (t de Student)

\begin{tabular}{|l|c|c|c|c|c|}
\hline & \multicolumn{4}{c|}{ Postest } \\
\hline Subescala & \multicolumn{4}{|c|}{ GT $=10)$ GC (N=11) } \\
\hline I: Autoexpresión en situaciones sociales & Media & SD & Media & SD & Sig. \\
\hline II: Defensa de los propios derechos como consumidor & 27,30 & 5,88 & 22,82 & 4,99 & SM \\
\hline III: Expresión de enfado o disconformidad & 18,50 & 2,01 & 12,64 & 4,63 & $* *$ \\
\hline IV: Decir no y cortar interacciones & 13,90 & 2,23 & 9,64 & 2,65 & $* *$ \\
\hline V: Hacer peticiones & 20,70 & 2,31 & 14,80 & 4,33 & $* *$ \\
\hline VI: Iniciar interacciones positivas con el sexo opuesto & 19,40 & 1,26 & 12,73 & 3,66 & $* *$ \\
\hline
\end{tabular}

NS: No significación.

* Significación menor o igual a 0,05.

* Significación menor o igual a o,OI.

SM: Significación Marginal menor a O, I y no menor de $0,05$.

Fuente: elaboración propia. 
En la tabla anterior, se observa un cambio en cada subescala después de ser aplicado el programa de entrenamiento. En este sentido, se obtienen diferencias estadísticamente significativas entre las respuestas de ambos grupos en las subescalas II, III, IV, V y VI. No obstante, sólo se encuentran diferencias a nivel marginal entre las respuestas de ambos grupos en la subescala I, referida a la "Autoexpresión en situaciones sociales".

- Análisis de las puntuaciones globales, pre y post GC y GT:

El mismo estadístico de contraste de medias, $t$ de Student, utilizamos para comparar las puntuaciones globales del GC y del GT antes
(Pre) y después (Post) de la implementación del programa de HHSS. Se pueden observan los resultados en las tablas 4,5 y y 5 b.

En relación con el Pre, se obtiene como resultado general que ambos grupos son equivalentes en las puntuaciones globales de la EAHS. No obstante, tras el entrenamiento se observan diferencias estadísticamente significativas en las puntuaciones medias de ambos grupos. Así, mientras que los participantes del GC mantuvieron puntuaciones globales en HHSS, similares en el Pre y en el Post, en el GT sí se observan cambios en la dirección esperada. Es decir, los participantes del GT mostraron un cambio positivo en sus puntuaciones después de ser aplicado el programa de entrenamiento en HHSS, con respecto al GC.

Tabla 4. Comparación de las medidas pre y postest de la puntuación global del GT y GC (t de Student)

\begin{tabular}{|l|c|c|c|c|}
\hline \multirow{2}{*}{} & \multicolumn{2}{|c|}{ Pretest } & \multicolumn{2}{c|}{ Postest } \\
\cline { 2 - 5 } & Grupo Tratamiento & Grupo Control & Grupo Tratamiento & Grupo Control \\
\cline { 2 - 5 } & $0_{1}$ & $0_{2}$ & $0_{3}$ & $0_{4}$ \\
\hline Puntuación global & 75 & 76,73 & 118,6 & 85,45 \\
\hline
\end{tabular}

Fuente: elaboración propia.

\begin{tabular}{l}
\hline Tabla 5a. Nivel de significación del pre-postest en los grupos GT-GC \\
\begin{tabular}{|l|c|c|}
\hline Contrastes de Medias & Pretest & Postest \\
\hline Grupo Tratamiento & $0_{1}$ & $\left.0_{3}{ }^{* *}\right)$ \\
\hline Grupo Control & $0_{2}$ & $0_{4}(\mathrm{NS})$ \\
\hline
\end{tabular}
\end{tabular}

Fuente: elaboración propia.

Tabla 5b. Nivel de significación de los grupos GT-GC en el pre y postest

\begin{tabular}{|l|c|c|}
\hline Contrastes de Medias & Grupo Tratamiento & Grupo Control \\
\hline Pretest & $0_{1}$ & $0_{2}(\mathrm{NS})$ \\
\hline Postest & $0_{3}$ & $\left.0_{4}{ }^{* *}\right)$ \\
\hline
\end{tabular}

NS: No significación.

* Significación menor o igual a 0,05 .

$*$ Significación menor o igual a 0,0 I.

Fuente: elaboración propia. 


\section{Discusión}

Los resultados obtenidos en el presente estudio sugieren que la implementación de un programa específico -ad hoc- de entrenamiento en HHSS dirigido a un grupo de personas adultas con DI, puede mejorar estas habilidades, las cuales resultan fundamentales para su desarrollo tanto personal como social. Más concretamente, podemos afirmar que el entrenamiento ha posibilitado un cambio positivo en la adquisición de HHSS y asertividad en el GT, confirmándose así la hipótesis general de la investigación, excepto para la dimensión "Autoexpresión en situaciones sociales".

Esto es valioso, al coincidir con autores como Verdugo y Gutiérrez Bermejo (2009), que sugieren cómo la aplicación de estos programas ayudan a prevenir dificultades en la adaptación y brindan estrategias clave para hacer frente a las diferentes situaciones que se les puedan presentar. Además, ayuda a favorecer el autoconcepto y la autoestima de las personas con discapacidad, influyendo así notablemente en su integración (Ojeda y Mateos, 2006). Estos datos guardan relación con lo expuesto por Zins et al. (I998), citado en Bremer y Smith (2004), que hacen hincapié en que este tipo de entrenamiento fomenta el desarrollo de relaciones auténticas y sinceras. Asimismo, favorece una integración y desinstitucionalización exitosa de la persona, facilitando la apertura a la comunidad (Gutiérrez Bermejo y Prieto, 2002) y fomentando la capacidad de autodeterminación de las personas con DI.

En la misma línea, O’Reilly et al. (2004) obtuvieron resultados positivos inmediatos tras la aplicación de un programa de HHSS a cinco adultos con DI, utilizando estrategias de resolución de problemas y de control externo. Además, todos mantuvieron las habilidades adquiridas cuatro semanas después de la intervención. Resultados similares hallaron Anderson y Kazantzis (2008) en un estudio de caso múltiple, donde tres adultos con DI participaron en un programa de I 5 sesiones de entrenamiento en resolución de problemas sociales. También el estudio de Liu et al. (2013), con datos pre-post, aunque sin grupo control, permite apoyar nuestros resultados. Así, ellos advierten de una mejora en habilidades sociales y de comunicación específicas para el contexto específico donde se había aplicado el programa a un grupo de I4 personas con DI y autismo, en su caso, el lugar de trabajo. Sin embargo, la mejora en otros comportamientos emocionales generales, tales como control de los impulsos, fue limitada.

De esta forma, podemos concluir que un programa específico -ad hoc- organizado sobre la base de las necesidades específicas previas detectadas en un grupo de adultos con DI puede proporcionar un cambio significativo en cuanto a la percepción que tienen los participantes sobre sus propias HHSS y su conducta asertiva. Ello se observa, principalmente, en los resultados obtenidos con la Escala Adaptada de Habilidades Sociales de Gismero, tras la aplicación del programa. Pero también podemos afirmar que, de forma cualitativa, se percibieron cambios positivos, claramente observables en el funcionamiento general de los participantes en el programa. Se observó por parte del grupo un mayor entendimiento de la importancia que tienen las competencias sociales en su día a día, fue característico después de la aplicación del programa un mayor uso de la asertividad por parte de los participantes, mostrando menos vergüenza a la hora de interactuar tanto con monitores como con otros compañeros.

En la misma línea, los resultados obtenidos por Lachavanne y Barisnikov (2013) ponen de manifiesto que además de observar mejorías significativas en las habilidades socioemocionales educadas, se produjo una reducción significativa de los problemas de conducta de los participantes. Por lo que sus resultados demostraron que los avances no se limitaban a este tipo de habilidades sino que también se habían generalizado a su nivel de funcionamiento diario. Este tipo de generalizaciones resulta esencial para fomentar el aprendizaje de destrezas sociales de forma constante en este grupo poblacional. 
También podemos hacer coincidir los resultados globales, es decir, el efecto positivo por parte del grupo que recibió el tratamiento (programa de HHSS), con los de Boluarte et al. (2006), donde advierten que la implementación de su programa de HHSS estaba relacionado con la mejora significativa de las habilidades de comunicación verbal y no verbal de jóvenes con DI leve y moderada.

Específicamente, en los resultados del análisis de las subescalas de la EAHS concluimos que no se hallaron diferencias estadísticamente significativas entre las respuestas de ambos grupos en el pretratamiento (Pre). Sin embargo, en el Post sí se obtienen diferencias entre los grupos GT y GC.

Fue en la subescala I "Autoexpresión en situaciones sociales” donde no se cumplieron exactamente las expectativas. De hecho, en dicha subescala ambos grupos alcanzaron las puntuaciones más altas, tanto antes (Pre) como después (Post) de la implementación del programa. Esto podría deberse a las características del entorno y del centro donde se realizó el presente estudio, al predominar un ambiente abierto, participativo, que brinda oportunidades de socialización entre sus usuarios.

Estos datos no coinciden exactamente con los obtenidos por Ojeda y Mateos (2006), donde se observa que las menores diferencias entre los grupos se consiguieron en aquellos elementos que pueden implicar más dificultades de interacción social, como "Iniciar interacciones con el otro sexo" y "Defender derechos como consumidor", subescalas VI y II, respectivamente. Los autores argumentaron que ello podía deberse a que estas habilidades podrían generar mayor dificultad en los usuarios, ya que se relacionan con aprendizajes y experiencias fuera de la familia. Fue la subescala III "Expresión de enfado o disconformidad" la que obtuvo la puntuación más baja en el presente estudio, tendiendo el grupo a evitar conflictos y confrontaciones con los demás.

En la misma línea, se presenta el estudio realizado por Martínez et al. (2004), en el que después de implementar un programa de entrenamiento en HHSS y de aplicar la misma Escala de Habilidades Sociales de Gismero a un grupo de diez pacientes psicóticos crónicos, los principales cambios se dieron en esta subescala III "Expresión de enfado o disconformidad", mejorando significativamente después de la intervención.

Quizás tales divergencias puedan explicarse, en gran medida, por las características diferenciales de las muestras, al contar en el presente estudio únicamente con personas con DI, mientras que el de Ojeda y Mateos (2006) estuvo formado por personas con distintas discapacidades, incluyendo sensoriales, físicas y cognitivas, y el de Martínez et al. (2004) con pacientes psicóticos. En consecuencia, podemos sugerir que un entrenamiento en HHSS puede arrojar diferentes resultados según el tipo de población al que va dirigido, por lo que sería recomendable que el entrenamiento de estas habilidades fuese específico y adaptado a las necesidades de cada población y contexto particular.

Así, como conclusión general con relación al Pre obtenemos que ambos grupos son equivalentes en las puntuaciones globales de la EAHS, mientras que en el Post debemos rechazar la hipótesis Ho; esto es, no podemos asumir que el GT haya respondido de igual manera después de completar el programa. Por tanto, si consideramos que durante el período de entrenamiento las condiciones (variables extrañas) se han mantenido constantes en ambos grupos, excepto en el entrenamiento realizado, podemos asumir razonablemente que la intervención ha posibilitado mejoras considerables en el GT en relación con los aspectos medidos en la EAHS. Así, estos resultados ponen de manifiesto que es posible lograr un cambio positivo en las personas con DI si se ejercitan las destrezas sociales necesarias para relacionarse eficientemente.

Esto lleva a plantearnos, como una de las recomendaciones, la importancia de que todos los centros que atienden a personas con DI y sus familias tengan instaurado en sus rutinas diarias el uso de habilidades sociales adecuadas. 
Autores como Umadevi y Sukumaran (2012) sugieren que el comportamiento social inapropiado de las personas con DI puede ser debido a una incorrecta percepción de la situación social. Además, de forma general, este tipo de intervenciones facilita el aprendizaje de estrategias de resolución de problemas y de toma de decisiones en las personas con DI.

Afortunadamente, en nuestro contexto contamos con propuestas de gran interés y relevancia para el entrenamiento de HHSS en personas con DI, cuando no existen programas específicos. Es el caso del Programa de Habilidades Sociales (PHS) de Verdugo (2006), que incluye seis áreas y 207 entrenamientos diferentes como conductas de integración y adaptación a la comunidad, habilidades de interacción social para establecer y mantener relaciones interpersonales eficaces, comunicación verbal para iniciar, mantener y finalizar conversaciones, desenvolvimiento independiente en la comunidad, ocio y tiempo libre, manejo del dinero o participación en actos sociales, entre otros. Otro interesante material lo ofrece la guía de trabajo para personas jóvenes y adultos con DI de diferentes niveles de gravedad de Gutiérrez Bermejo y Prieto (2002), que permite planificar el entrenamiento en HHSS a través de protocolos de trabajo específicos y habilidades como: contacto ocular, distancia interpersonal, escucha activa, seguimiento de instrucciones, pedir y prestar ayuda, expresar sentimientos, etc. Más recientemente, Gutiérrez Bermejo (20I0) realiza una propuesta más específica centrada en el entrenamiento en habilidades socio-sexuales en jóvenes y adultos con DI, para evitar que sean víctimas o perpetradores de abusos sexuales.

Por otro lado, entre las limitaciones encontradas en el presente estudio, destacamos el reducido tamaño de la muestra, lo que supone un hándicap para la generalización de los resultados, y el difícil acceso a la familia, que dificultó la obtención de información sobre el comportamiento de las personas en su hogar, dinámicas familiares, etc.

Para futuros estudios, además de subsanar las limitaciones señaladas, sería deseable la utilización de una escala específica para la evaluación de las HHSS en personas con DI, antes y después del entrenamiento, como por ejemplo la Escala de actuación social revisada, que actualmente están desarrollando Arias et al. (20I4). La ampliación del número de sesiones del programa, para entrenar con más profundidad algunos de los objetivos y la inclusión de un período de seguimiento no inferior a cuatro semanas, con sesiones de refuerzo.

Por otro lado, como medida para impulsar el enfoque inclusivo en el tratamiento de la diversidad, se podrían extraer aquellos aspectos del programa de entrenamiento de habilidades sociales aplicado que fueran extrapolables a otras discapacidades.

Finalmente, con todo lo mencionado podemos asumir que el entrenamiento ha posibilitado mejoras en el grupo de usuarios que participó en el programa (GT) en relación con los aspectos medidos en la EAHS, considerándose de esta manera que el entrenamiento en HHSS favorece el incremento de estas habilidades. Por tanto, dados los resultados obtenidos en nuestro estudio y en otros similares, se recomienda promocionar el diseño de programas específicos - ad hoc- para mejorar la competencia social en los contextos interpersonales significativos para los adultos con DI.

No obstante, cuando esto no sea posible, se debería optar por la aplicación de programas genéricos como los sugeridos más arriba, al menos en los centros de día que atienden a población adulta con DI.

Además, aunque no se llevaron a cabo análisis estadísticos confirmatorios, el seguimiento y el trabajo diario con los usuarios que participaron en este programa nos ha permitido constatar que los resultados positivos alcanzados se han mantenido a lo largo del tiempo. De esta forma, en todos los casos, se han realizado evaluaciones posteriores sobre diferentes dominios y competencias de carácter psicosocial, para actualizar documentación personal. En dichas valoraciones, hemos observado indicadores que ponen de manifiesto un rendimiento en el área de habilidades sociales superior al recogido en 
usuarios que nunca han recibido entrenamiento, así como mejores puntuaciones en comparación con los datos disponibles de los participantes en otros momentos temporales. Adicionalmente, se ha observado una mayor implicación de dichos usuarios en actividades de la comunidad, adoptando un rol activo, siendo capaces de ajustarse adecuadamente a diferentes contextos y resolviendo dificultades de forma funcional. Resultados que justifican ampliamente que sigamos aplicando programas específicos para el entrenamiento de habilidades sociales a nuestros usuarios.
La frase "Nadie sabe de lo que es capaz hasta que lo intenta" de Publio Siro es reflejo de lo que se pretende transmitir en el presente trabajo, resaltando la importancia que tiene brindar a las personas con discapacidad los recursos necesarios para su desarrollo. Esto incluye el fomento del uso de unas habilidades sociales adecuadas que les permitan vincularse de forma efectiva en la sociedad, no como meros receptores sino como agentes activos de la misma. 
Anexo I. Ejemplos de ítems de la Adaptación de la Escala de Habilidades Sociales (EHS)

Autor: Elena Gismero González.

Adaptada por: Fernanda Sequera.

Ejemplos de ítems adaptados
14. Cuando tengo mucha prisa y me llama un amigo por
teléfono, me cuesta mucho cortarlo.
Ejemplo: Si estoy trabajando y un amigo me habla, me
cuesta parar la conversación.
15. Hay determinadas cosas que me disgusta prestar, pero
si me las piden, no sé cómo negarme.
Ejemplo: Si un amigo me pide prestado mi móvil, no sé
cómo decirle que no.
32. Muchas veces prefiero ceder, callarme o quitarme de en
medio para evitar problemas con otras personas.
Ejemplo: Si discuto con algún amigo, prefiero callarme y no
decir nada, para evitar problemas.

Fuente: elaboración propia con el permiso concedido por la Dirección del Departamento I+D+i Research \& Development Manager para la inclusión de estos tres ítems a modo ilustrativo (fecha: 23/05/20I6). 
AAIDD (20I I): Discapacidad Intelectual: Definición, Clasificación y Sistemas de Apoyo, Madrid: Alianza Editorial.

Anderson, G. y Kazantzis, N. (2008): "Social problem-solving skills training for adults with mild intellectual disability: A multiple case study". Behavior Change, 25 (2): 97-108.

Arias, V. B. et al. (20I4): "Evaluación de las habilidades sociales en adultos con discapacidad intelectual: calibración de la Escala SPSS-R mediante el modelo de Rasch". Behavioral Psychology/Psicología Conductual, 22 (3): 479-500.

Bellack, A. S. ( 1983 ): "Recurrent problems in the behavioral assessment of social skill". Behavioral Research and Therapy, 2I: 29-4I.

Bielecki, J. y Swender, L. S. (2004): “The assessment of social functioning in individuals with mental retardation". Behaviour Modification, 28: 694-708.

Boluarte, A. et al. (2006): "Programa de Entrenamiento en Habilidades Sociales para Jóvenes con Retraso Mental Leve y Moderado". Mosaico Cient, 3 (I): 34-42.

Bremer, C. y Smith, J. (2004): “Teaching Social Skills". National Center on Secondary Education and Transition. Information Brief, 3 (5): I-5.

Caballo, V. E. (I993): Manual de evaluación y entrenamiento de las habilidades sociales, Madrid: Siglo XXI.

Elksnin, N. y Elksnin, L. (200I): “Adolescents with disabilities: the need for occupational social skills training”. Exceptionality, 9: 9I-IO5.

Gismero, E. (2010): Escala de Habilidades Sociales. Manual, Madrid: TEA, S.A.

Gutiérrez Bermejo, B. (2010): Habilidades Sociosexuales en Personas con Discapacidad Intelectual, Madrid: Pirámide.

Gutiérrez Bermejo, B. y Prieto, M. (2002): Manual de Evaluación y Entrenamiento en Habilidades
Sociales para Personas con Retraso Mental, Junta de Castilla y León: Consejería de Sanidad y Bienestar Social. Gerencia de Servicios Sociales.

Hair, E. et al. (2002): Helping Teens Develop Healthy Social Skills and Relationships: What Research Shows About Navigating Adolescence, Washington, DC: Child Trends.

Kelly, J. A. (2002): Entrenamiento de las habilidades sociales, Bilbao: Desclée De Brouwer.

Lachavanne, A. y Barisnikov, K. (20I3): "Rééducation des compétences socioémotionnelles pour des adultes présentant une déficience intellectuelle". European Review of Applied Psychology, 63 (6): 345-352.

León, O. G. y Montero, I. (I997): Diseño de Investigación. Introducción a la Lógica de la Investigación en Psicología y Educación, Madrid: McGraw-Hill.

Liu, K. P. et al. (20I3): "Effectiveness of a Workplace Training Programme in Improving Social, Communication and Emotional Skills for Adults with Autism and Intellectual Disability in Hong Kong- A Pilot Study”. Occupational Therapy International, 20: 198-204.

Martínez, J. et al. (2004): "Habilidades Sociales en Pacientes Psicóticos Crónicos”. Interpsiquis, 5: I-I3 .

Matson, J. L. y Boisjoli, J. A. (2008): “Cutoff scores for the Matson Evaluation of Social Skills for Individuals with Severe Retardation (MESSIER) for adults with intellectual disability". Behavior Modification, 32 (I): 109I 20 .

Morales, P. (2013): Investigación experimental, diseños y contraste de medias (en línea). <http://www.academia.edu/6859778/ Investigaci $\% \mathrm{C}_{3} \% \mathrm{~B}_{3} \mathrm{n} \_$experimental_ Investigaci $\% \mathrm{C}_{3} \% \mathrm{~B}_{3} \mathrm{n} \_$experimental_ dise $\% \mathrm{C}_{3} \%$ B Ios_y_contraste_de_medias $>$, acceso I 8 de febrero de 2016. 
O’Reilly, M. F. et al. (2004). "Teaching social skills to adults with intellectual disabilities: a comparison of external control and problem-solving interventions". Research in Developmental Disabilities, 25: 399-4I2.

Ojeda, B. y Mateos I. (2006): "Habilidades Sociales y Satisfacción Familiar en Sujetos con Discapacidad”. Rehabilitación Psicosocial, 3 (I): $2-8$.

Roca, E. (2007): Cómo mejorar tus Habilidades Sociales. Programa de Asertividad, Autoestima e Inteligencia Emocional, Valencia: ACDE Psicología.

Rosales, J. J. et al. (20I3): Habilidades sociales, Madrid: McGraw-Hill.

Ruiz, E. (2007): "Programa de Educación Emocional para Niños y Jóvenes con Síndrome de Down”. Revista Síndrome de Down, 2: 84-93.

Shaughnessy J. et al. (2007): Métodos de Investigación en Psicología, México, DF: McGraw-Hill.

Umadevi, V. y Sukumaran, P. (20I2): "Functional Social Skills of Adults with Intellectual
Disability". Disability, CBR and Inclusive Development, 23 (2): 72-80.

Verdugo, M. A. (2006): Programa de Habilidades Sociales (PHS). Programas Conductuales Alternativos, Salamanca: Amarú.

Verdugo, M. A., y Gutiérrez Bermejo, B. (2009): Discapacidad Intelectual. Adaptación y Problemas de Comportamiento, Madrid: Pirámide.

Wilkins, J. y Matson, J. L. (2009): “A Comparison of Social Skills Profiles in Intellectually

Disabled Adults With and Without ASD". Behavior Modification, 33 (2): I43-I 55.

VV.AA. (2010): PAP. Protocolo de Atención Personalizada para Personas con Discapacidad Usuarias de Servicios Residenciales y de Atención Diurna, Sevilla: Consejería para la Igualdad y Bienestar Social.

Zins, J. E. et al. (1998): "Enhancing Learning through Social and Emotional Education". Think: The Journal of Creative and Critical Thinking, 9: I8-20. 